over 8 years old, and/or carers. Completion of forms was voluntary and anonymised. In 2019 all respondents were offered the opportunity to take part in semi-structured interviews to explore their experiences in detail. Interviewees completed a questionnaire prior to the interview where they ranked the elements they most valued with regards to urgent care visits. This was used to scaffold the interview. Data was collected by 2 researchers, who transcribed verbatim, and by participants' written reports.

In 2019 data was collected over a three-week period. All data collection and analyses were carried out by individuals independent from the clinical team. Qualitative data was thematically analysed by four researchers. This was the second part of an audit cycle.

Results In 201951 (18 involving patients) responses to the PREM questionnaire were collected and 12 interviews (4 involving patients) took place (20-30 minutes long).

The majority described ED experience positively. From the PREM questionnaire key strengths included addressing pain in a timely manner and safety netting. Areas that need improvement include updates when waiting and advice on when to resume normal activities.

From the interviews, communication and safety were identified as priorities. The provision of detailed information in a timely manner was highly valued, alongside feeling listened to by staff. Safety had three dimensions: physical, clinical and psychological/emotional. Waiting times and environmental factors also were valued but were less than other factors above.

Conclusions Communication is highly valued, but improvements are required to provide contemporaneous information. This is congruent with existing literature highlighting parent disempowerment in acute care settings and underlining the importance of timely updates. Good communication improved carers' feelings of psychological and emotional safety.

\section{G144(P) SERVICE EVALUATION OF EMERGENCY ORAL AND MAXILLOFACIAL SURGICAL CARE OF PAEDIATRIC PATIENTS REQUIRING GENERAL ANAESTHESIA}

K Rajendran, A Dickenson. Oral and Maxillofacial Surgery, Royal Derby Hospital, Derby, UK

\subsection{6/archdischild-2020-rcpch.115}

Background Children who require emergency surgery should receive effective and high-quality management in their local hospital. |

Aims The primary aim of this service evaluation was to determine the standard of our emergency surgical care of paediatric patients requiring GA.

Objectives To determine the standard of our emergency surgical care of paediatric patients requiring GA; To assess the number of paediatric OMFS emergency admissions that required surgery under GA; To assess if the treatment received under GA was appropriate and to review if the time period between when the patient presented to $\operatorname{CED}\left(\mathrm{T}_{0}\right)$ and when the patient was taken to theatre $\left(\mathrm{T}_{\mathrm{T}}\right)$.

Method Case records of 43 patients under 18 years of age who required non-elective OMFS surgery between Jan 2014 to Dec 2017 were analysed.

Results $44 \%$ of the emergency admissions were as a result of acute dental infections. Dental trauma and facial bones fractures were seen more in males than females. The average age for emergency incision and drainage of dental abscess under GA was 7 years.

Discussion $100 \%$ of surgical interventions performed on patients admitted to secondary care were appropriate. As acute dental infections resulted in $44 \%$ of hospital admissions, this highlights that caries in children is a significant public health problem and poses a significant burden on the limited NHS resources.

Recommendations Discuss at departmental clinical governance meeting any reasons cited as delay to surgery and implement any changes to protocol. Develop a written local protocol regarding admission of emergency OMFS paediatric patients to inform new staff of the process to improve efficiency and thereby enhance service provision.

\section{G145(P) REVIEW OF THE MANAGEMENT OF CHILDREN WITH MASTOIDITIS: A SINGLE CENTRE EXPERIENCE}

${ }^{1} \mathrm{~S}$ Islam, ${ }^{1} \mathrm{~T}$ Chowdhury, ${ }^{2} \mathrm{~V}$ Alexander, ${ }^{3} \mathrm{SB}$ Drysdale. 'Faculty of Medicine, St George's Hospital Medical School, London, UK; ${ }^{2}$ Department of Ear, Nose and Throat, St George's Hospitals NHS FT, London, UK; ${ }^{3}$ Department of Paediatrics, St George's Hospitals NHS FT, London, UK

\subsection{6/archdischild-2020-rcpch.116}

Aims Mastoiditis has an incidence of $\sim 8.2 / 100,000$ hospital admissions, though rare it can cause intracranial complications. The Trust's antibiotic policy recommends co-amoxiclav as first line treatment and ceftriaxone plus metronidazole if there are concerns about concurrent intracranial infection.

We aimed to review the diagnostic features, management and outcome of children with acute mastoiditis.

Methods Children receiving antibiotics were identified from the Trust's antimicrobial database. We retrospectively analysed the notes of children admitted between 01/01/17 and 10/05/ 19 with an initial diagnosis of mastoiditis. We identified 25 patients in whom a diagnosis of acute mastoiditis was made on hospital presentation. We reviewed their notes to identify presenting symptoms, investigations undertaken and medical and surgical management.

Results 25 patients were initially suspected of mastoiditis, diagnosis was confirmed in 16 (64\%). Alternative diagnoses included otitis media $(n=4,16 \%)$, otitis externa $(n=3$, $12 \%)$, cholesteatoma $(n=1,4 \%)$ and pre-auricular lymphadenopathy $(n=1,4 \%)$. Patients with a confirmed diagnosis of mastoiditis had a mean (range) age of 4.5 (0.1-12) years and mean (SD) duration of hospitalisation of 3.6 (2.0) days. Post-auricular swelling was seen in 69\% $(n=11)$, displacement of pinna in $56 \%$ and mastoid erythema in $56 \%$ $(n=9) .31 \%(n=5)$ were treated with antibiotics by their GP prior to admission. Mean CRP on admission was 113.9 (SD 103.4) $\mathrm{mg} / \mathrm{L}$ and mean white cell count $15.5 \times 10^{9} / \mathrm{L}(\mathrm{SD}$ $\left.4.4 \times 10^{9} / \mathrm{L}\right) .37 \%(\mathrm{n}=6)$ had a head CT scan. No patient had confirmed intracranial infection. 69\% $(n=11)$ received IV co-amoxiclav as first line management, the remaining $31 \%(n=5)$ ceftriaxone and metronidazole. Mean (SD) duration of inpatient antibiotics was 3.6 (2.1) days and outpatient antibiotics $12.7(9.9)$ days. 31\% $(n=5)$ had surgical intervention; only one $(6 \%)$ child had positive microbiology (Streptococcus pneumoniae from pus). 81\% ( $\mathrm{n}=13)$ had an ENT follow up appointment. 